\title{
Dimensión vertical oclusal en niños de 3, 4 y 5 años con relación a su biotipo facial
}

\author{
Relationship between the vertical occlusal dimension and facial biotype \\ in 3, 4 and 5 year-old children
}

Martha Araujo Ramirez ${ }^{\text {1ad }}$

Jhon Mezarina Mendoza ${ }^{1 a b c}$

iD https://orcid.org/0000-0002-2004-4938

(iD https://orcid.org/0000-0002-3496-2502

Correspondencia: vanessaaraujo218@gmail.com

\section{Resumen}

Objetivo: Determinar la relación existente entre la dimensión vertical oclusal y el biotipo facial en los niños de 3, 4 y 5 años de la institución educativa inicial N. ${ }^{\circ} 591$ Carlos Manuel Cox durante el año 2019. Métodos: El estudio utilizó un diseño no experimental y transversal. La muestra fue realizada a través de un muestreo no probabilístico por casos consecutivos y estuvo comprendida por 62 niños de 3,4 y 5 años de la I.E.I. N. ${ }^{\circ} 591$ Carlos Manuel Cox, que cumplieron con los criterios de inclusión y exclusión. Los niños fueron examinados en posición de máxima intercuspidación, registrando las medidas del punto subnasal a mentoniano, altura facial (ofrion-gnation) y ancho facial (distancia bicigomática) mediante un calibrador digital. Posteriormente se realizó el análisis estadístico en el programa SPSS v21. Resultados: Se encontró que la dimensión vertical en promedio fue de $54.11 \pm 2.99 \mathrm{~mm}$. Los niños con biotipo dolicofacial presentaron una mayor dimensión vertical oclusal con un valor de $55.45 \pm 2.80 \mathrm{~mm}$. Se evidenció que la relación entre el biotipo facial y las medidas de la dimensión vertical oclusal fueron estadísticamente significativas $(p=0.006)$. Conclusión: Existe relación entre el biotipo facial y la dimensión vertical oclusal, lo que indica que distintas formas faciales presentan características asociadas a la longitud del tercio inferior facial.

Palabras clave: dimensión vertical, biotipo facial, niños

\begin{abstract}
Objective: To determine the relationship between the vertical occlusal dimension and the facial biotype in 3 , 4 and 5 year-old children of the kindergarten N. ${ }^{\circ} 591$ Carlos Manuel Cox, in 2019. Methods: The study used a non-experimental and cross-sectional design. The sample was carried out through a non-probabilistic sampling for consecutive cases and was comprised of 62 children who met the inclusion and exclusion criteria. The children were examined in a position of maximum intercuspation, recording the measurements of the subnasal to chin point, facial height (ofrion-gnation) and facial width (bizygomatic distance) using a digital caliper. The statistical analysis was performed in the SPSS v21 program. Results: The average vertical dimension was found to be $54.11 \pm 2.99 \mathrm{~mm}$. Children with a dolichofacial biotype had a greater occlusal vertical dimension with a value of $55.45 \pm 2.80 \mathrm{~mm}$. It was evidenced that the relationship between the facial biotype and the measurements of the occlusal vertical dimension were statistically significant $(p=$ 0.006). Conclusion: There is a relationship between the facial biotype and the vertical occlusal dimension, which indicates that different facial shapes have characteristics associated with the length of the lower third of the face.
\end{abstract}

Keywords: vertical dimension, facial biotype, children

${ }^{1}$ Universidad Nacional Mayor de San Marcos. Facultad de Odontología. Lima, Perú

${ }^{a}$ Cirujano Dentista

${ }^{\mathrm{b}}$ Esp. en Odontopediatría

- Mg. en Gerencia de los Servicios de la Salud

${ }^{d}$ Egresada de la Maestría en Estomatología

Fecha de recepción: 09/02/2021

Fecha de aceptación: 08/04/2021 


\section{Introducción}

Durante el transcurso de la niñez, la integridad de la dentición decidua se ve afectada por distintas enfermedades bucales como la caries dental, las lesiones pulpares y traumatismos que, muchas veces, no son tratadas oportunamente, dando como resultado la pérdida prematura de dientes deciduos. Con el paso del tiempo, esto ocasiona una serie de alteraciones como, por ejemplo, deficiencias en el desarrollo y crecimiento de los maxilares, alteraciones en la cronología y secuencia eruptiva dentaria, dificultades en el lenguaje, habla y la autoestima. ${ }^{1}$

Las distintas enfermedades bucales pueden alterar el perfil facial y las proporciones maxilomandibulares como la dimensión vertical oclusal (DVO), que es considerada como un punto determinante para que se logre un equilibrio a nivel oclusal y facial. Cuando la DVO se altera puede causar afecciones a nivel oclusal, alteraciones musculares y articulares, pudiendo desencadenar en disfunciones temporomandibulares, además de comprometer la armonía funcional y estética. ${ }^{2,3}$

Diversos autores, como Zúñiga S., Andronic A. y Matamala V., realizaron estudios sobre la pérdida prematura de dientes deciduos, donde coinciden que los dientes más afectados son los molares temporales; siendo, justamente, la pérdida de estos dientes los principales responsables de la alteración de la DVO. ${ }^{4-6}$

Según el glosario de términos prostodónticos (2017), la DVO está definida como la "distancia entre dos puntos anatómicos o marcados seleccionados (usualmente un punto está en la base de la nariz y otro en el mentón) cuando los maxilares están en posición de máxima intercuspidación".7 Además, la alteración de la DVO está asociada generalmente con la pérdida de varios dientes o el desgaste severo de estos. Esta situación produce alteraciones en el sistema estomatológico, como disfunción temporomandibular, disfunción masticatoria, alteración en la fonética, alteración en la musculatura y falta de estética dental. ${ }^{8,9}$ Algunos estudios también asocian la pérdida de dimensión vertical con problemas neuromusculares, propioceptivos y posturales. ${ }^{10,11}$

Por su parte, el biotipo facial se define como un conjunto de caracteres funcionales y morfológicos que guían el crecimiento y el comportamiento funcional del rostro de un individuo; relacionados entre sí, así se den por herencia o trastornos funcionales. ${ }^{12,13}$ Está clasificado por tres tipos básicos, según la relación entre los componentes del rostro (horizontal y vertical), medidos en la distancia bicigomática y desde el punto nasión hasta el mentón. ${ }^{14}$

Pérez et al. estudiaron las alteraciones dimensionales de la DVO en niños de 5 a 7 años, concluyendo que existe una ligera disminución de la DVO desde los 6 hasta los 6 años y medio, lo que se puede deber al cambio de dentición que existe durante esa etapa. ${ }^{15}$

Ferreira señaló que es imprescindible un registro adecuado de la DVO para poder realizar un tratamiento rehabilitador exitoso, tomando en cuenta la distinción por grupo de edad y sexo, ya que alteraciones en la DVO pueden generar problemas a nivel oclusal, muscular, articular y predisposiciones a la instalación de maloclusiones. ${ }^{16}$

Barrios indicó que el uso de prótesis en infantes edéntulos contribuye a reestablecer la función a nivel masticatorio, fonético, estético y a mejorar en el nivel psicológico. De esta manera se puede lograr un desarrollo saludable y un adecuado nivel de erupción para las piezas permanentes, por lo que se sugirió el uso de prótesis como tratamiento para pacientes infantes edéntulos parciales y totales. $^{17}$

La odontopediatría ofrece diversas opciones de tratamientos rehabilitadores para el restablecimiento funcional y estético de la oclusión. La rehabilitación oral en niños que han perdido 
los dientes por caries de infancia temprana, traumatismos, alteraciones o síndromes que presenten ausencias múltiples de piezas dentarias es muy compleja, ya que no se tienen valores promedios de DVO de acuerdo al biotipo facial para poder rehabilitar satisfactoriamente a los pacientes niños. Además, en niños con fisuras labiopalatinas, las prótesis son una valiosa ayuda para la correcta pronunciación de los sonidos y mejorar la apariencia física.

En el grupo pediátrico, el análisis de la DVO se torna aún más relevante, porque estos pacientes se encuentran en un crecimiento constante y todas las estructuras articulares, bases óseas y apicales se encuentran en desarrollo. No obstante, existen muy pocos estudios en niños para obtener medidas clínicas promedio de la DVO por grupo etario específico, por ello el establecer medidas guía es fundamental para ejecutar la rehabilitación adecuada de la DVO, respetando los parámetros de normalidad. ${ }^{18-20}$

El presente estudio tuvo como limitación el reducido número de muestra, pero esto se debe a los criterios de inclusión de la investigación, recordemos que, según los reportes del Minsa (2017), ${ }^{26}$ la enfermedad de caries dental es del $60 \%$, sumado a esto, los niños del presente estudio no debieron tener ningún tratamiento preventivo ni restaurativo, por lo que la muestra se reduce significativamente.

El presente estudio tuvo como objetivo determinar un valor promedio de DVO en la población pediátrica, en grupo etario de 3, 4 y 5 años de edad, relacionándolo al biotipo facial que presente cada niño.

\section{Método y materiales}

El estudio utilizó un diseño no experimental, de tipo observacional, transversal. Los criterios de inclusión fueron los siguientes: (a) niños de 3,4 y 5 años de edad con dentición decidua completa y normoclusión o maloclusión Clase I, sin lesiones de caries cavitadas; (b) que los tutores o padres hayan firmado el consentimiento informado; (c) el asentimiento de los niños.

Se excluyeron a los pacientes con anomalías de forma, número y estructura dentaria; con molares permanentes erupcionados; con restauraciones preventivas y/o correctivas; con tratamiento ortodóntico u ortopédico maxilar, actual o pasado; portadores de síndromes; niños de los cuales sus padres y/o tutores no hayan firmado el consentimiento informado y niños que no den su asentimiento. ${ }^{21}$

La población del estudio comprendió a los niños de la I.E.I. N. ${ }^{\circ} 591$ Carlos Manuel Cox. La muestra fue seleccionada por un muestreo no probabilístico por casos consecutivos, la cual estuvo compuesta por 62 niños de 3, 4 y 5 años que cumplieron con los criterios de inclusión.

Se solicitó el permiso respectivo a la I.E.I. N. 591 Carlos Manuel Cox para poder realizar el estudio. Previo a la recolección de la muestra se realizó un proceso de calibración, en donde mediante el índice de Kappa se obtuvo un valor de 1.

Para la toma de los registros se procedió a ubicar al niño en una silla, sentado en una posición de $90^{\circ}$, con la cabeza alineada en relación al cuerpo, la vista fija en un punto determinado y bajo luz natural. Se procedió a ubicar los puntos cefalométricos desde la base de la nariz, punto subnasal (Sn) hasta la parte inferior del mentón, punto mentoniano (Me). Para su mejor identificación y medición se marcó estos puntos. Para realizar las mediciones se utilizó un vernier electrónico Mitutoyo Americano Absolute (Figura 1). 


\section{Figura 1}

Registro de medidas de dimension vertical y biotipo facial

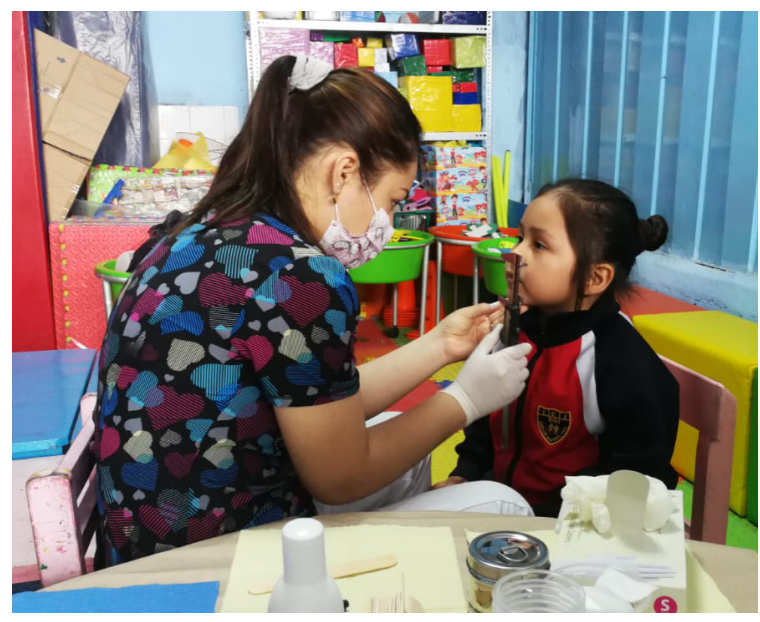

Todas las medidas se tomaron en máxima intercuspidación y se registraron en milímetros. Para determinar el biotipo facial se utilizó el índice facial morfológico total; donde la altura facial es la distancia entre el ofrion y el gnation, y el ancho facial está representado por la distancia bicigomática en el tejido blando. De acuerdo a los resultados hallados con la fórmula, estos se categorizaron de la siguiente manera: un índice facial mayor a 104 corresponde a un tipo facial dolicofacial, de 97 a 104 se consideró mesofacial y menor a 97, braquifacial. ${ }^{22,23}$

Los resultados fueron anotados en una ficha de recolección; luego se procedió a valorarlos de acuerdo a los criterios correspondientes para determinar la medida de la DVO y su respectivo biotipo facial. Los datos se procesaron de manera automatizada utilizando el programa SPSS v21. En el análisis descriptivo de las variables cualitativas se utilizaron tablas de frecuencias. Para el análisis descriptivo de las variables cuantitativas se utilizaron medidas de tendencia central y dispersión. La correlación entre la dimensión vertical oclusal y el biotipo facial se realizó a través del análisis de correlación de Spearman. Para comparar los datos entre variables categóricas y numéricas se utilizaron tablas de contingencia y un análisis de homogeneidad, según la prueba Anova.

\section{Resultados}

Se evaluó a 62 niños, donde el $54.8 \%$ fueron del sexo masculino y el $45.2 \%$ del sexo femenino. El promedio de dimensión vertical oclusal (DVO) obtenido en la muestra fue de $54.11 \pm 2.99 \mathrm{~mm}$, en donde se encontró que el sexo femenino tuvo mayor promedio de DVO, siendo este de $54.29 \pm 3.20$ $\mathrm{mm}$, mientras que el sexo masculino obtuvo un promedio menor de $53.93 \pm 2.47 \mathrm{~mm}$.

La dimensión vertical oclusal (DVO) promedio del grupo de 5 años fue mayor con respecto a los grupos de 3 y 4 años (Tabla 1 ).

Tabla 1

Dimensión vertical oclusal según edad

\begin{tabular}{ccccc}
\hline DVO & 3 años & 4 años & 5 años & Total \\
\hline X \pm DE & $52.78 \pm 3.24 \mathrm{~mm}$ & $54.36 \pm 2.74 \mathrm{~mm}$ & $55.22 \pm 2.64 \mathrm{~mm}$ & $54.11 \pm 2.99 \mathrm{~mm}$ \\
Rango & $11.02 \mathrm{~mm}$ & $12.35 \mathrm{~mm}$ & $10.46 \mathrm{~mm}$ & $13.89 \mathrm{~mm}$ \\
IC 95\% & $51.22-54.34 \mathrm{~mm}$ & $53.25-55.46 \mathrm{~mm}$ & $53.86-56.58 \mathrm{~mm}$ & $53.35-54.87 \mathrm{~mm}$
\end{tabular}

DVO= Dimensión vertical oclusal; $\mathrm{X}=$ Media; $\mathrm{DE}=$ Desviación estándar; $\mathrm{IC}=$ Intervalo de confianza 
Se encontró menor cantidad de niños con biotipo braquifacial (22.8\%), siendo el biotipo dolicofacial más frecuente entre ellos (41.9\%). Además, se evidenció que, en grupo de 3 y 5 años, predominó el biotipo dolicofacial $(16.1 \%$ y $11.3 \%$, respectivamente), mientras que en el de 4 años predominó el biotipo mesofacial (16.1\%) (Figura 2).

\section{Figura 2}

Biotipo facial según edad

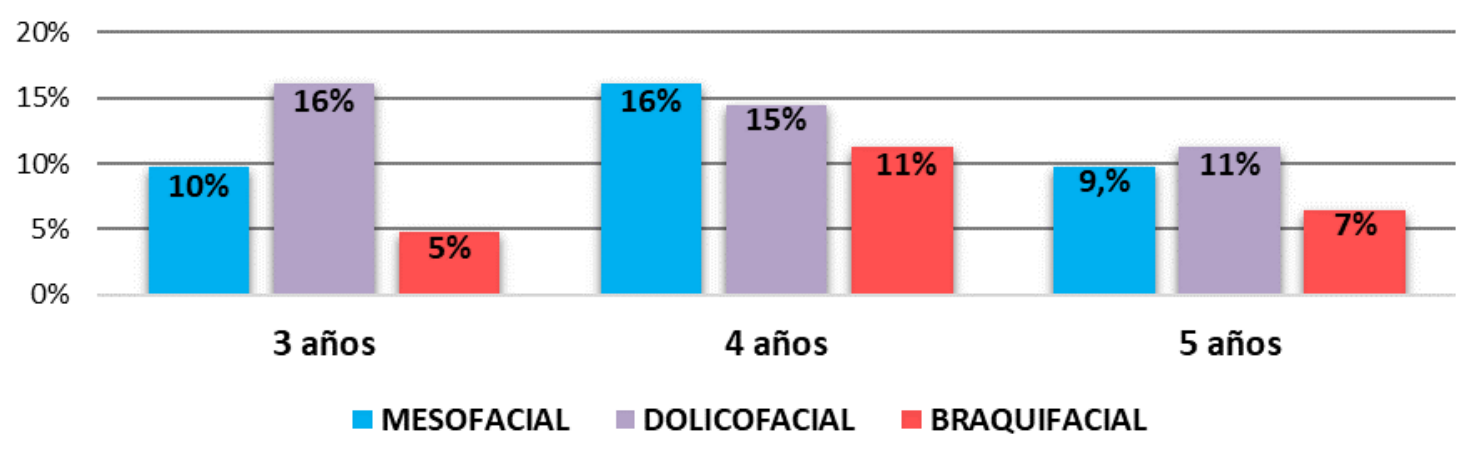

En el sexo femenino se encontró mayor cantidad de niños con biotipo dolicofacial, mientras que en el sexo masculino el porcentaje fue igual para el biotipo dolicofacial y mesofacial (19.4 \%) (Figura 3).

\section{Figura 3}

Biotipo facial según sexo

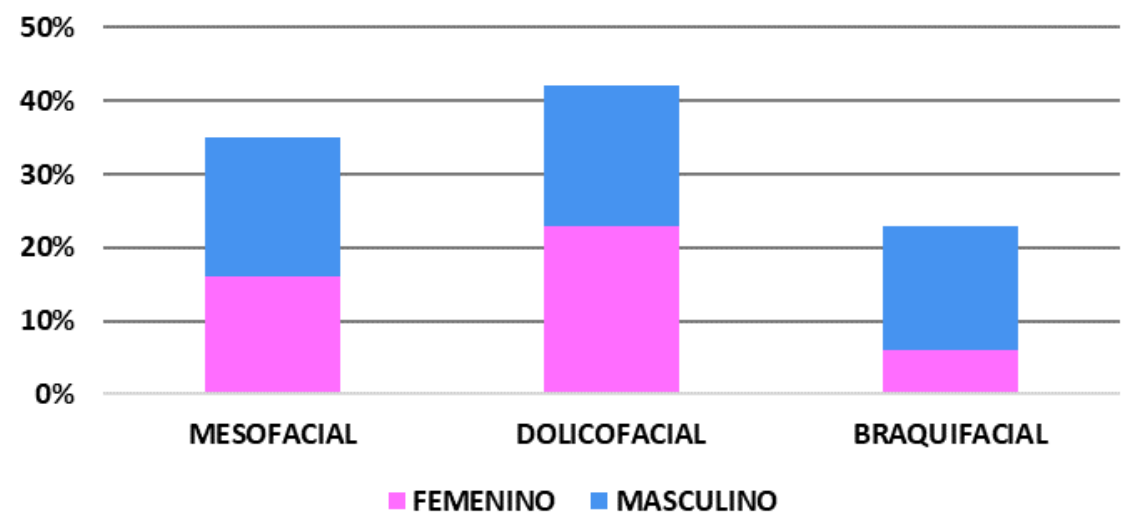

Al evaluar la DVO, según cada biotipo facial, se encontró que el biotipo dolicofacial presentó un promedio mayor de DVO, siendo este valor de $55.45 \mathrm{~mm}$; en donde el grupo de 5 años obtuvo una media mayor de $56.76 \mathrm{~mm}$. Por el contrario, el biotipo braquifacial presentó el menor promedio de DVO, siendo este de $52.58 \mathrm{~mm}$, en donde el grupo de 4 años obtuvo una media mayor de 53.56 $\mathrm{mm}$ (Tabla 2).

\section{Tabla 2}

Dimensión vertical oclusal según biotipo facial yedad

\begin{tabular}{ccccc}
\hline Biotipo Facial & 3 años & 4 años & 5 años & Total \\
\hline Mesofacial & $50.34 \pm 2.94 \mathrm{~mm}$ & $54.10 \pm 2.28 \mathrm{~mm}$ & $55.63 \pm 1.84 \mathrm{~mm}$ & $53.49 \pm 3.07 \mathrm{~mm}$ \\
Dolicofacial & $54.71 \pm 2.24 \mathrm{~mm}$ & $55.26 \pm 3.82 \mathrm{~mm}$ & $56.76 \pm 1.62 \mathrm{~mm}$ & $55.45 \pm 2.80 \mathrm{~mm}$ \\
Braquifacial & $51.24 \pm 3.19 \mathrm{~mm}$ & $53.56 \pm 1.41 \mathrm{~mm}$ & $51.89 \pm 2.38 \mathrm{~mm}$ & $52.58 \pm 2.21 \mathrm{~mm}$ \\
\hline
\end{tabular}


En la muestra evaluada se encontró que sí existe relación entre dimensión vertical y biotipo facial $(p=0.015 ; r=0.349)$, siendo esta relación de manera directa. Al comparar las medidas de dimensión vertical oclusal se encontró que existen diferencias significativas entre los distintos biotipos faciales $(p=0.006)$. Además, se encontró relación entre las muestras con biotipo mesofacial y dolicofacial $(p=0.015)$, dolicofacial y braquifacial $(p=0.000)$; sin embargo, entre las muestras de mesofacial y braquifacial no se encontró relación $(p=0.210)$.

Al evaluar la DVO y el sexo de la muestra se halló que no existe relación entre ellos $(p=0.406)$.

Finalmente, se evidenció que sí existe relación entre la DVO y la edad de cada paciente $(p=0.041)$, evidenciándose relación entre los grupos de edades de 3 con 5 años $(p=0.037)$, mas no con los grupos de 3 con 4 años $(p=0.175)$, ni 4 con 5 años $(p=0.607)$.

\section{Discusión}

En población pediátrica que ha perdido prematuramente piezas dentarias, la determinación de una DVO correcta es uno de los puntos clave para lograr este objetivo; sin embargo, ningún método de obtención de esta medida es considerado totalmente correcto en niños, es por esto que el presente estudio buscó determinar un promedio de DVO en niños de 3, 4 y 5 años con dentición decidua según la armonía facial que presentase cada niño.

Cardoso evaluó la dimensión vertical oclusal mediante el método craneométrico de Knebelman en una población peruana con relación a su biotipo facial, en donde se evidenció que sí existe relación en población adulta. El presente estudio lo evaluó mediante medidas clínicas en población pediátrica, en donde se encontró que dicha relación también es existente en niños. ${ }^{24}$

En el estudio también se evidenció una distancia mayor de DVO en el sexo femenino (54.29 mm) en comparación con el sexo masculino $(53.93 \mathrm{~mm})$, contrarrestando con los estudios realizados por Matta y Sagawa en los cuales se observa una medida mayor de DVO en varones que en mujeres. Esto puede deberse a que en el presente estudio se obtuvo una muestra mayor de niños con biotipo dolicofacial y del sexo femenino. ${ }^{25}$

El presente estudio tuvo como limitación el reducido número de la muestra, pero esto se debe a los criterios de inclusión de la investigación, recordemos que, según los reportes del Minsa (2017), ${ }^{26}$ la enfermedad de caries dental es del $60 \%$, sumado a esto, que los niños del presente estudio no debieron tener ningún tratamiento preventivo ni restaurativo, por lo que la muestra se redujo significativamente.

Se puede indicar que la relación encontrada servirá para la guía de posteriores procedimientos clínicos en el área de odontopediatría, como lo son las rehabilitaciones en niños edéntulos parciales o totales, de esta manera se logrará mejorar la función masticatoria, la estética y fonética del niño, brindándole una vida social idónea que le permita desarrollarse adecuadamente en su entorno. Además, favorecerá el desarrollo de los músculos masticatorios y periorales, en consecuencia, el patrón de crecimiento de los huesos basales.

Se recomienda realizar más trabajos de investigación con una muestra mayor y de distintos departamentos para poder extrapolar estas referencias a otras latitudes, ya que actualmente existe escasa cantidad de antecedentes referidos a niños, puesto que es un tema aún poco conocido en la literatura odontopediátrica.

\section{Conclusiones}

Se encontró relación directa entre las medidas de la dimensión vertical oclusal y el biotipo facial. Al comparar las categorías de biotipo facial se encontró relación entre las muestras con biotipo mesofacial-dolicofacial y dolicofacial-braquifacial; sin embargo, entre las muestras de mesofacialbraquifacial no se encontró relación.

Se evidenció relación entre dimensión vertical oclusal y edad, mas no con el sexo. 


\section{Referencias}

1. Segura N, Gutiérrez M, Ochoa M, Díaz J. Pérdida prematura de dientes temporales y maloclusión en escolares. Correo Médico Científico Holguín. 2005; 9(3).

2. Chacona RL. Enamel loss and occlusal vertical dimension: causes and considerations for treatment. Dent Today. 2003; 22(4):92-7.

3. Mack MR. Vertical dimension: a dynamic concept based on facial form and oropharyngeal function. J Prosthet Dent. 1991; 66(4):478-85.

4. Zúñiga S. Prevalencia de pérdida prematura de dientes temporales en niños 3-9 años. [Tesis de bachillerato]. [Guayaquil]: Facultad de Ciencias Médicas, Universidad Católica de Santiago de Guayaquil; 2017.

5. Andronic A. Prevalence of early loss of primary teeth in 6-10 year old school children in Sibiu. Clinica Aspects. 2017; 22(4): 128-9

6. Matamala CV. Prevalencia y factores de riesgo relacionados con pérdidas prematuras de dientes temporales en escolares de 4 a 7 años. Comuna de Talcahuano 2016. [Tesis de bachillerato]. [Chile]: Facultad de Odontología, Universidad Andrés Bello; 2016.

7. Ferro KJ. The Academy of Prosthodontics. The glossary of prosthodontic terms. 9th ed. J Prosthet Dent. 2017; 117(5): 50-90.

8. Cawood JI, Howell RA. A classification of the edentulous jaws. Int J Oral Maxillofac Surg. 1988;17: 232(6)-236.

9. García-Fajardo C, Cacho A, Fonte A, Pérez JC. La oclusión como factor etiopatológico en los trastornos temporomandibulares. RCOE. 2007; 12 (1-2): 37-47.

10. Marcé M. Prótesis Completa: Técnica Cortada. A propósito de un caso. Dentum. 2004; 4 (2): 55-61.

11. Lundstrom A. Introducción a la ortodoncia. Editorial Mundi. Argentina. 1960.

12. Gregoret J, Tuber E, Escobar LH, \& Matos da Fonseca A. Ortodoncia y Cirugía Ortognática. Diagnóstico y planificación. 2. ${ }^{\circ}$ Ed. Barcelona: Editorial Espaxs; 2008.

13. Arriaga EL. Relación clase esquelética y patrón facial. Oral. 2000; 3, 50-2.

14. Allen PF, Jepson NJ, Doughty J. Attitudes and practice in the provision of removable partial dentures. Br Dent J. 2008; 204.

15. Pérez N, Chelotti A, Piloto M, Mirabal J. Estudio longitudinal de la dimensión vertical de oclusión, en niños cubanos, de 5 a 7 años de edad. Rev. cubana ortod. 2001;16(1):54-8.

16. Ferreira A. Hermida L. Santos K. Mesquita R. Domingues M. Franco L. Rodrigues T. Kalil S. Evaluación de la variabilidad de la dimensión vertical de oclusión en niños y adolescentes. Actas odontológicas. 2011; VIII (1):37 42.

17. Barrios Z, Salas M. Prótesis en dentición primaria: Revisión de la literatura. Rev. Odont. de los Andes. 2006; 1(2): 61-9.

18. Geerts GA, Stuhlinger ME, Nel DG. A comparison of the accuracy of two methods used by predoctoral students to measure vertical dimension. J Prosthet Dent. 2004; 91(1):59-66.

19. Misch CE. Guidelines for maxillary incisal edge position-a pilot study: the key is the canine. J Prosthodont. 2008; 17(2):130-4.

20. Cutbirth ST. Increasing vertical dimension: considerations and steps in reconstruction of the severely worn dentition. Pract Proced Aesthet Dent. 2008; 20(10):619-26.

21. Hernández S, Fernández C, Baptista P. Metodología de la investigación. México DF: Mc Graw Hill; 2010.

22. Mayoral J, Mayoral G. Ortodoncia. Principios fundamentales y prácticos. 6ta. ed. Barcelona: Labor; 1990. 79.

23. Proffit W. The etiology of the orthodontic problems. In: Proffit WR, Fields HW, eds. Contemporary Orthodontic. 3ed. St. Louis: Mosby; 2000: 13-144.

24. Cardoso SL. Evaluación de la dimensión vertical oclusal mediante el método craneométrico de Knebelman en una población peruana con relación a su biotipo facial. [Tesis de bachillerato]. [Lima]: Facultad de Odontología, UNMSM; 2014. 75p.

25. Matta C, Sagawa J. Comparación entre la zona facial media y el tercio facial inferior en 
estudiantes de 19 a 25 años de edad de la Facultad de Estomatología de la UPCH. Rev. Estomatología Herediana 2003; 13(1-2):23-6.

26. Guía de práctica clínica para la prevención, diagnóstico y tratamiento de la caries dental en niñas y niños: Guía técnica. Ministerio de Salud. Dirección General de Intervenciones Estrategias en Salud Pública. Dirección de Salud Bucal. Lima: Ministerio de Salud; 2017.

El presente artículo deriva de una tesis de pregrado publicada en el siguiente link: https://hdl.handle.net/20.500.12672/11242

- Conflicto de intereses: La presente investigación no presenta conflicto de intereses entre los investigadores.

- Fuente de financiamiento: La presente investigación fue financiada por los investigadores. 